\title{
Emergent Adaptive Noise Reduction From Communal Cooperation of Sensor Grid
}

\author{
Kennie H. Jones \\ NASA Langley Research Center \\ Hampton, VA, U.S.A. \\ k.h.jones@nasa.gov \\ Michael G. Jones \\ NASA Langley Research Center \\ Hampton, VA, U.S.A. \\ michael.g.jones@nasa.gov
}

\author{
Douglas M. Nark \\ NASA Langley Research Center \\ Hampton, VA, U.S.A. \\ d.m.nark@nasa.gov \\ Kenneth N. Lodding \\ NASA Langley Research Center \\ Hampton, VA, U.S.A. \\ kenneth.n.lodding@nasa.gov
}

\begin{abstract}
In the last decade, the realization of small, inexpensive, and powerful devices with sensors, computers, and wireless communication has promised the development of massive sized sensor networks with dense deployments over large areas capable of high fidelity situational assessments. However, most management models have been based on centralized control and research has concentrated on methods for passing data from sensor devices to the central controller. Most implementations have been small but, as it is not scalable, this methodology is insufficient for massive deployments. Here, a specific application of a large sensor network for adaptive noise reduction demonstrates a new paradigm where communities of sensor/computer devices assess local conditions and make local decisions from which emerges a global behaviour. This approach obviates many of the problems of centralized control as it is not prone to single point of failure and is more scalable, efficient, robust, and fault tolerant.
\end{abstract}

Keywords: sensor networks, adaptive noise reduction, emergent behavior, community, autonomous, cooperation, situation awareness

\section{Introduction}

The realization of sensor networks was first demonstrated in 1999 by the Smart Dust project [1] when small, inexpensive devices, or motes, that could sense, compute and communicate wirelessly were deployed over a geographic region. Although the Smart Dust project produced a small, working mote, their deployments were limited to small numbers of motes collecting information and forwarding it through a multi-hop network to a central controller: a sense and send model. In 2000, the National Research Council published a call for research funding [2] predicting that sensor networks would soon facilitate pervasive computing where sensor and actuator nodes would be everywhere in the environment enabling complete, automated control of electro-mechanical processes.

Jones et al. [3] documented that, in the following years, little progress has been made to achieve this goal. Most working implementations are limited to less than 50 sensor devices with simulations limited to less than 300 . Few implementations include actuators and most implementations use the sense and send model. It is not clear that techniques developed will scale to massively sized networks. By its architecture, the sense and send model is limited in size and function. As the size of the grid increases, the multi-hop network forces nodes closest to the central controller to expend energy for routing leading to energy exhaustion long before outer nodes. Centralized control is vulnerable to single point of failure and cannot achieve the sophisticated applications predicted.

Jones et al. [4] demonstrated an alternate approach where motes are viewed not as nodes in a computer network but as autonomous, yet cooperating, entities much like organisms in an ecosystem. Their situational awareness is limited to a locality and no one ever knows all global information. Their actions are also local and based only on local information. From the culmination of all of these local actions, global behavior emerges. This approach mitigates many of the problems of centralized control and the sense and send method.

In the following sections, this approach is applied to the problem of adaptive noise reduction in aircraft engine nacelles. It will be shown that optimal noise attenuation emerges when communities of sensor/actuator motes cooperate to determine local impedance settings based on local situational awareness. Section 2 provides background on the current state of noise reduction in aircraft engine nacelles and the advantages that could be provided by adaptive noise control. Section 3 details the specifications of the model tested and the simulation techniques used. Section 4 describes the algorithm for determining impedance values for optimal noise attenuation using local situational awareness. Section 5 describes the simulation and presents results. Finally, concluding remarks are offered in Section 6. 


\section{Background on Noise Reduction in Aircraft Engine Nacelles}

Noise reduction is currently achieved in aircraft engine nacelles by a set of homogeneous, static and passive Helmholtz resonators [5]. The inside of the nacelle is lined with a metallic sheet with thousands of small holes. These holes cover numerous Helmholtz resonators that consist of a honeycomb core of, for the most part, uniform depth. Hence, the impedance spectrum (impedance is a frequency-dependent parameter) of the liner is nearly constant for a selected flight regime; i.e., a uniform liner. Due to nonlinear effects, the impedance spectrum changes as the flight regime changes. Thus, the liner must be designed as a compromise among the multiple flight regimes, such that it provides an acceptable level of noise attenuation throughout all flight regimes.

Although current implementations have uniform liners, research has shown that noise attenuation can be increased by liners in which the resonators are divided into segments of differing impedance: a segmented liner. Mani [6] and Watson [7] proved segmented liners can be designed to significantly outperform the best uniform liner for a given acoustic source.

Recently, an active Helmholtz resonator has been designed that can adjust its impedance [8]. Changing the impedance will change the resonator's ability to attenuate noise. The challenge is to determine the optimal impedance for a given acoustic source.

The goal here is to develop and analyze a replacement for the current static and passive liner of aircraft engine nacelles by an active, intelligent, and adaptive system that can adjust its impedance to account for changing acoustic conditions resulting from changes in the operational environment. The architecture employed uses a community of intelligent and autonomous, yet cooperative, resonators instead of the more common hierarchical, centralized design approach. An implementation of dynamically tunable resonators to provide an optimal liner could be implemented by a central controller, which could respond to a changing input source by lookup of the optimum liner impedance from a predetermined table. However, an algorithm has been developed in which the individual liner resonators cooperate with their local neighbors to derive, dynamically and in situ, their own impedance tuning based on local information to improve sound reduction. The challenge was to produce a rule set for making this decision from which a maximum global attenuation emerges: engineering for desirable emergent behavior.

To assess noise reduction, a metric is required. Noise attenuation results from a reduction in sound pressure exiting from the nacelle. Sound pressure is measured in decibels (dB), a logarithmic scale. Thus, a 10-fold increase in pressure $(\mathrm{P})$ results in an increase of $20 \mathrm{~dB}(20$ $\log _{10} \mathrm{P}$ ), and a doubling of pressure results in an increase of $\sim 6 \mathrm{~dB}$. Therefore, an increase of a few $\mathrm{dB}$ in attenuation is a significant reduction in noise.

\section{Model Specifications and Simulation Techniques}

The geometry considered in this analysis is a rectangular duct with a 15 " X 6 " cross section and an axial length of 32". As depicted in Fig. 1, one entire wall of the duct (15" $\mathrm{X} 32$ ") is acoustically treated (the liner) along the full extent, while the other three sides are hardwall. The liner may be uniform or segmented. The acoustic source input consisted of the least attenuated mode at frequencies varying from 1000 to $2500 \mathrm{~Hz}$ in $500 \mathrm{~Hz}$ increments. These frequencies were chosen to provide a variety of aeroacoustic conditions requiring different optimal impedances (i.e., an impedance combination providing optimal attenuation at one frequency generally will not perform as well at other frequencies).

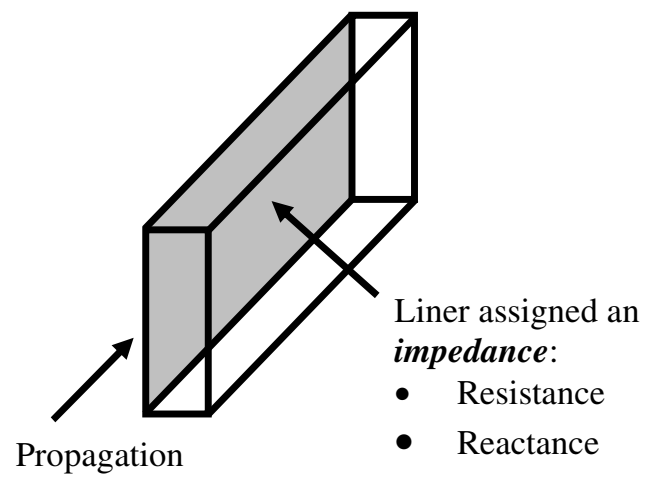

Fig. 1. Rectangular duct for analysis, 15" x 6"

The analysis software used to perform the in-duct acoustic propagation calculation and overall attenuation prediction for a given liner was CDUCT-LaRC [9], designed to study propagation within and radiation from a three-dimensional geometry such as the duct described above. Given a computational grid, mean flow solution, acoustic source, and, optionally, an acoustic liner positioned somewhere at the boundaries of the duct, CDUCT-LaRC will calculate the propagation within the duct geometry. Various pre and post processors were developed to prepare input to CDUCT-LaRC, determine impedance choices for analysis following the algorithm described in the next section, and analyze output.

\section{Algorithm for Selecting Liner Segment Impedance}

Impedance is represented by a complex number where the real component is the resistance and the imaginary component is reactance. The domain of "allowable" impedance values $I$ can be represented as an $\mathrm{R}_{\mathrm{s}} \mathrm{X} \mathrm{\textrm {R } _ { \mathrm { a } }}$ matrix with $R_{s}$ possible resistance values and $R_{a}$ possible reactance values. Obviously, the overall dimensions of this impedance domain are a function of the range of resistance and reactance values to be considered, as well as the relative spacing chosen between subsequent values. For a given frequency, the uniform liner providing highest 
attenuation can be determined by executing CDUCTLaRC once for each impedance in the domain $I$. For the current investigation, the impedance domain $I$ was composed of resistance values in the range, [0.1 .. 2.5], and reactance values in the range, $\left[\begin{array}{lll}-2.0 & .2 & 2.0\end{array}\right]$, each incremented by 0.1 . Fig. 2 displays a subset of the domain $I$ for $1000 \mathrm{~Hz}$. The maximum attenuation for this domain of $42.7 \mathrm{~dB}$ is at $I(0.9,-0.8)$ attained with a uniform liner of impedance $=0.9-0.8 \mathrm{i}$.

\section{Ra}

$\begin{array}{llllllllll}-1.3 & -1.2 & -1.1 & -1 & -0.9 & -0.8 & -0.7 & -0.6 & -0.5\end{array}$

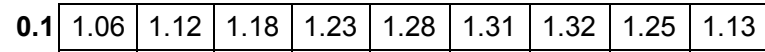

\begin{tabular}{|l|l|l|l|l|l|l|l|l|l|}
$\mathbf{0 . 2}$ & 3.00 & 3.20 & 3.39 & 3.56 & 3.69 & 3.75 & 3.72 & 3.60 & 3.39 \\
\hline
\end{tabular}

\begin{tabular}{|l|l|l|l|l|l|l|l|l|l|l|}
$\mathbf{0 . 3}$ & 4.93 & 5.29 & 5.63 & 5.3 & 6.16 & 6.27 & 6.22 & 6.02 & 5.69 \\
\hline
\end{tabular}

\begin{tabular}{|l|l|l|l|l|l|l|l|l|l|l|}
$\mathbf{0 . 4}$ & 6.86 & 7.40 & 7.92 & 8.39 & 8.76 & 8.94 & 8.89 & 8.59 & 8.09 \\
\hline
\end{tabular}

\begin{tabular}{|l|l|l|l|l|l|l|l|l|l|l|}
$\mathbf{0 . 5}$ & 20.36 & 9.51 & 10.26 & 10.96 & 11.54 & 11.86 & 11.83 & 11.38 & 10.61 \\
\hline
\end{tabular}

\begin{tabular}{|l|l|l|l|l|l|l|l|l|l|}
$\mathbf{0} .6$ & 22.30 & 27.00 & 12.64 & 13.65 & 14.56 & 15.17 & 15.21 & 14.52 & 13.31 \\
\hline
\end{tabular}

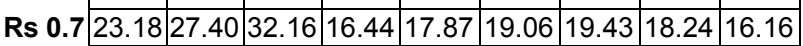

\begin{tabular}{|l|l|l|l|l|l|l|l|l|l|}
0.8 & 23.26 & 26.97 & 31.19 & 36.00 & 41.96 & 23.66 & 25.73 & 22.74 & 18.86 \\
\hline
\end{tabular}

\begin{tabular}{|l|l|l|l|l|l|l|l|l|l|}
\hline 0.9 & 22.78 & 25.94 & 29.52 & 33.75 & 39.52 & 42.70 & 33.54 & 25.50 & 20.59 \\
\hline
\end{tabular}

\begin{tabular}{|l|l|l|l|l|l|l|l|l|l|}
1 & 21.90 & 24.48 & 27.24 & 30.13 & 32.69 & 32.90 & 29.39 & 24.77 & 20.87 \\
\hline
\end{tabular}

\begin{tabular}{|l|l|l|l|l|l|l|l|l|l|}
\hline 1.1 & 20.79 & 22.81 & 24.82 & 26.62 & 27.76 & 27.55 & 25.71 & 22.98 & 20.20 \\
\hline
\end{tabular}

\begin{tabular}{|l|l|l|l|l|l|l|l|l|l|}
1.2 & 19.58 & 21.13 & 22.56 & 23.70 & 24.27 & 24.01 & 22.87 & 21.11 & 19.13 \\
\hline
\end{tabular}

\begin{tabular}{|l|l|l|l|l|l|l|l|l|l|}
1.3 & 18.38 & 19.57 & 20.60 & 21.34 & 21.65 & 21.42 & 20.63 & 19.42 & 17.99 \\
\hline
\end{tabular}

Fig. 2. Attenuation values for uniform liners of impedance values in domain $I$.

As displayed in Table 4, an impedance neighborhood in the domain is defined as matrix element $I\left(\mathrm{R}_{\mathrm{s}}, \mathrm{R}_{\mathrm{a}}\right)$ and its eight adjacent elements:

Table 4: A neighborhood in the impedance domain $I$.

\begin{tabular}{|c|c|c|}
\hline$I\left(\mathrm{R}_{\mathrm{s}}-0.1, \mathrm{R}_{\mathrm{a}}-0.1\right)$ & $I\left(\mathrm{R}_{\mathrm{s}}-0.1, \mathrm{R}_{\mathrm{a}}\right)$ & $I\left(\mathrm{R}_{\mathrm{s}}-0.1, \mathrm{R}_{\mathrm{a}}+0.1\right)$ \\
\hline$I\left(\mathrm{R}_{\mathrm{s}}, \mathrm{R}_{\mathrm{a}}-0.1\right)$ & & \\
\hline & $I\left(\mathrm{R}_{\mathrm{S}}, \mathrm{R}_{\mathrm{a}}\right)$ & $I\left(\mathrm{R}_{\mathrm{S}}, \mathrm{R}_{\mathrm{a}}+0.1\right)$ \\
$I\left(\mathrm{R}_{\mathrm{S}}+0.1, \mathrm{R}_{\mathrm{a}}-0.1\right)$ & $I\left(\mathrm{R}_{\mathrm{S}}+0.1, \mathrm{R}_{\mathrm{a}}\right)$ & $I\left(\mathrm{R}_{\mathrm{S}}+0.1, \mathrm{R}_{\mathrm{a}}+0.1\right)$ \\
\hline
\end{tabular}

Notice, in Fig. 2, that, for impedance producing maximum attenuation at $I(0.9,-0.8)$, all neighbors have less attenuation. Also, for any other impedance, there is at least one neighboring impedance with greater attenuation and only one neighbor impedance with the greatest attenuation in the neighborhood. The algorithm for determining the entry with highest attenuation for the domain is as follows:

1. Given an initial impedance, examine the attenuation produced by all impedances in its impedance neighborhood.

2. Select the neighbor producing the highest attenuation and make that the center of a new impedance neighborhood. (Hereafter steps 1 and 2 are referenced as a neighborhood assessment).
3. Continue this process until the impedance of the neighborhood's center entry produces the highest attenuation for the neighborhood. This terminates the process as it has identified the entry producing the highest attenuation in the domain.

A simulation was performed assessing global attenuation and adjusting the impedance of a uniform liner using this algorithm. For each input frequency, the algorithm terminated at the impedance producing optimal attenuation for the uniform liner. However, this approach can never achieve better attenuation than that of the best uniform liner.

Rather than evaluating global attenuation, a better approach is to evaluate local power within the duct to assess changes in impedance. This would reduce computation and communication costs, as well as distribute the decision process. As will be shown, from a combination of proper assessments of local power resulting in changes to the impedance, the optimal global attenuation will emerge.

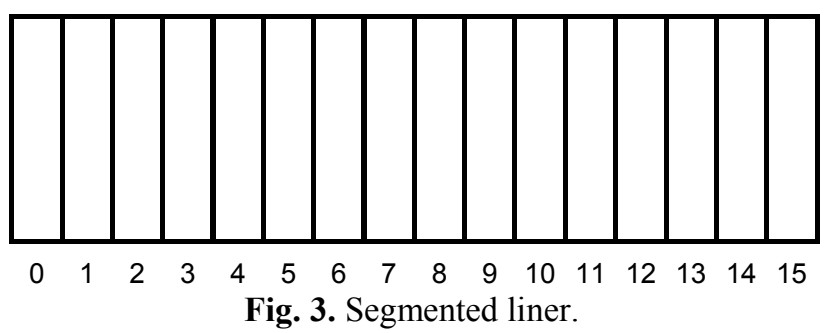

Depicted in Fig. 3, the liner is divided into segments axially with $\mathrm{S}_{0}$ at the duct entrance (source location) and $\mathrm{S}_{15}$ at the duct exit. A leader segment is selected and all segments towards the exit from the leader serve as its slaves. That is, the leader decides on impedance changes and all resonators within that segment and the resonators of all slave segments adapt to that decision. Initially, segment $\mathrm{S}_{0}$ is assigned as the leader. It executes a loop performing a series of neighborhood assessments: examining all neighbors, selecting the best impedance, selecting a new neighborhood based on this best impedance, and terminating when the selected impedance is better than any of its neighbors. With each decision, it dictates to its resonators and to the resonators of all slave segments the new impedance. All of these resonators tune to this impedance. When the leader terminates, it freezes its impedance for the remainder of the process and turns leadership over to its adjacent segment towards the exit. This process continues until segment $\mathrm{S}_{14}$ terminates. Segment $S_{15}$ assigns to its resonators the best impedance determined by the termination of segment $S_{14}$. Leadership is returned to the segment $\mathrm{S}_{0}$ and the process terminates. All segments retain their current impedance until segment $\mathrm{S}_{0}$ determines that the local assessment has dropped below a threshold, and the process begins again. The result of this process is a segmented liner producing a significantly increased global attenuation. 
It is important to note that while the initial leader assignment requires communication from opposing ends of the liner, this is not a requirement for global information: there are still only two segments providing information for a decision. With each transfer of leadership, the distance between the two opposing, communicating segments decreases.

Instead of a global attenuation assessment, this approach looks at a local assessment: the change in acoustic power level. To determine the optimal impedance, the leader segment $S_{L}$ obtains its own acoustic power level $\mathrm{PWL}_{\mathrm{L}}$, obtains segment $\mathrm{S}_{15}$ 's acoustic power level $\mathrm{PWL}_{15}$, and computes its delta power level, $\triangle \mathrm{PWL}$, as:

$$
\Delta \mathrm{PWL}_{\mathrm{L}}=10 * \log _{10}\left(\mathrm{PWL}_{\mathrm{L}} / \mathrm{PWL}_{15}\right)
$$

The leader segment sets its impedance in turn to each within the current neighborhood of impedances. It computes the delta power level at each impedance and selects the impedance producing the highest delta power level. It sets itself to this impedance which forms the center of a new impedance neighborhood and dictates that each slave segment also set their resonators to this impedance. This process continues until the leader segment finds the impedance producing the highest $\triangle \mathrm{PWL}$. At this point, the leader segment freezes its impedance and turns over leadership to the adjacent segment towards the exit. Each leader segment executes the above loop until segment $\mathrm{S}_{14}$, as leader, freezes its impedance and dictates this impedance to segment $S_{15}$.

\section{Simulation of Adaptation to Best Segmented Liner}

The simulation that follows represents this scenario. The initial frequency is set to $1500 \mathrm{~Hz}$. A uniform liner is arbitrarily set to impedance $=1.1-1.1 i$, which yields a relatively low attenuation for the initial frequency, 14.40 $\mathrm{dB}$. The liner will adapt following the algorithm described in Section 4 until the highest attenuation emerges and holds that liner definition until the frequency changes. As the frequency changes to $1000 \mathrm{~Hz}$, the attenuation decreases. The liner then adapts until the highest attenuation emerges and holds that liner definition until the frequency changes. This process repeats for the frequencies, $2000 \mathrm{~Hz}$ and $2500 \mathrm{~Hz}$. For all frequencies, logical neighborhoods and communal cooperation are used to adapt to a segmented liner that produces higher attenuation than the best uniform liner.

Fig. 4 depicts the final impedance assignments for $1500 \mathrm{~Hz}$. At the top of the figure is the liner divided into 16 segments. The bottom of the figure depicts the impedance matrix and the values assumed by each segment as it freezes. With $\mathrm{S}_{0}$ as leader, the impedance producing the highest $\Delta \mathrm{PWL}$ is $1.3-1.1 i$ (marked by the index 1). When all segments are set to this impedance, the liner is uniform and produces the highest attenuation for a uniform liner at $25.01 \mathrm{~dB}$. However, when $\mathrm{S}_{1}$ completes its leadership role, it freezes at impedance, 1.3-1.3i (marked by index 2) and the global attenuation is higher than that of the best uniform liner at $31.92 \mathrm{~dB}$. As leadership is forwarded, subsequent segments freeze at other (though possibly the same) impedances resulting in the map in Fig. 4 and the final global attenuation of 33.5 $\mathrm{dB}$.

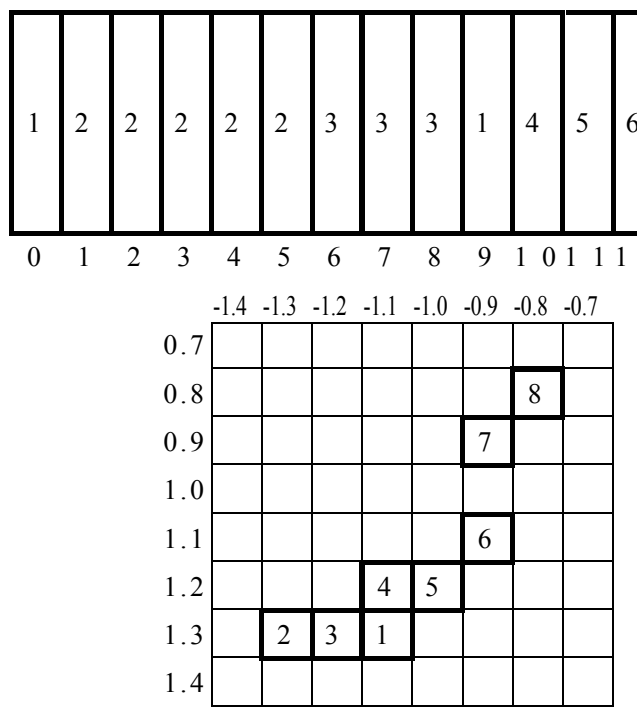

Fig. 4. Final segmented liner for $1500 \mathrm{~Hz}$. Global attenuation $=33.5 \mathrm{~dB}$.

What is truly remarkable is the unusual distribution of impedances throughout the liner. While this arrangement produces the highest attenuation, it is not readily apparent how such an arrangement was determined. Yet the community of sensor motes determine this arrangement without any global knowledge: the arrangement emerges from the combination of their local actions.

Fig. 5 shows the global attenuation increase as the simulation progresses and each leader segment freezes at the best impedance. Notice that the attenuation quickly exceeds the best for any uniform liner. 


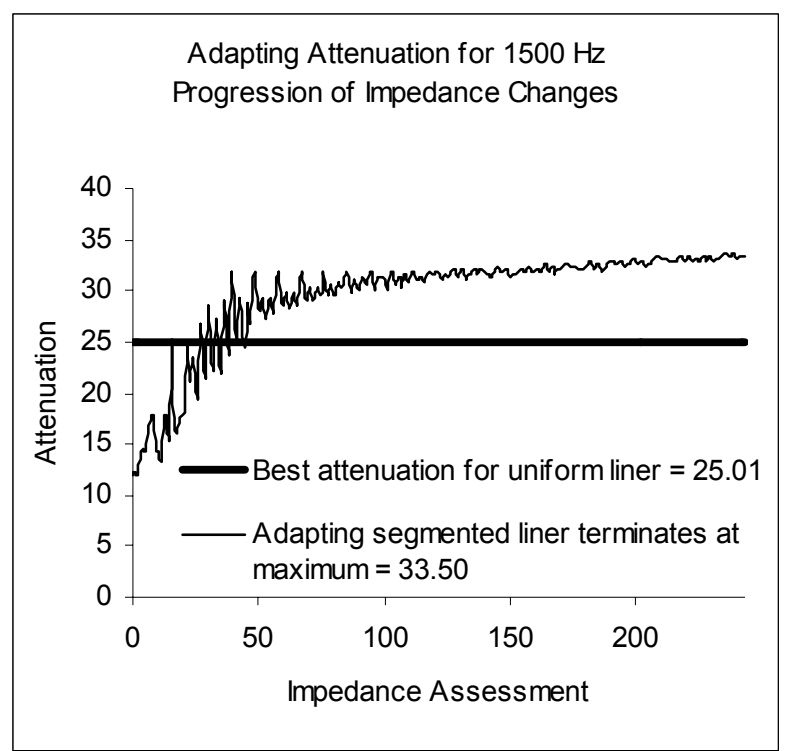

Fig. 5. Adaptation progression for segmented liner at $1500 \mathrm{~Hz}$.
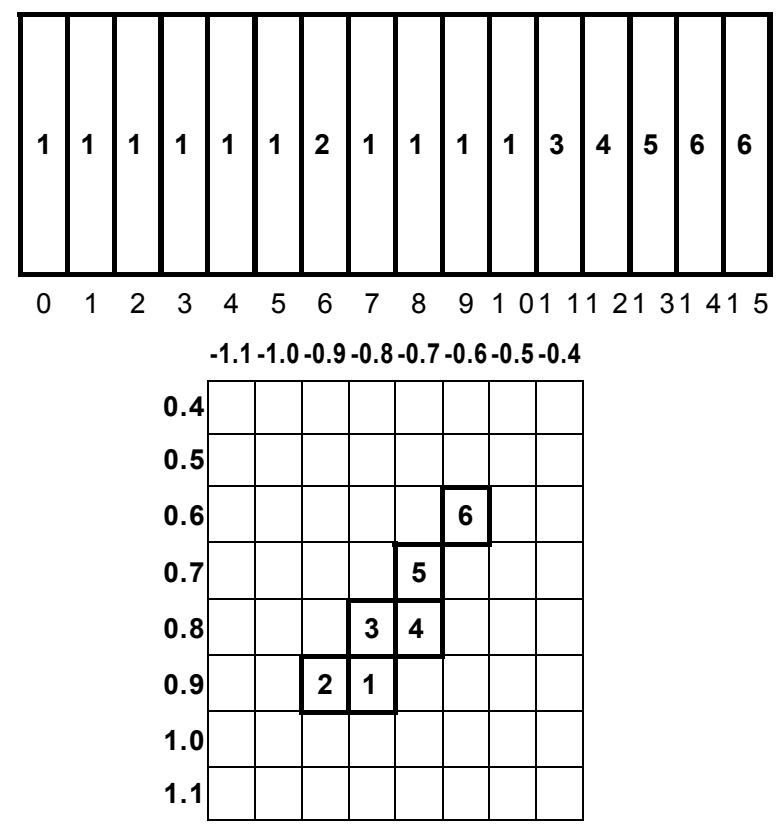

Fig. 6. Final segmented liner for $1000 \mathrm{~Hz}$. Global attenuation $=46.57 \mathrm{~dB}$.

Figs. 6 and 7 show the results of the simulation for $1000 \mathrm{~Hz}$. Notice that, unlike $1500 \mathrm{~Hz}$., the impedance remains constant for segments $0-5$ and $7-10$, but remaining segments are of different impedances.

Fig. 7 compares the adapting attenuation to that of the best attenuation for a uniform liner for $1000 \mathrm{~Hz}$. Notice that, while it takes longer to adapt to an attenuation better than that of the best uniform liner, it does exceed it by a significant amount before terminating.

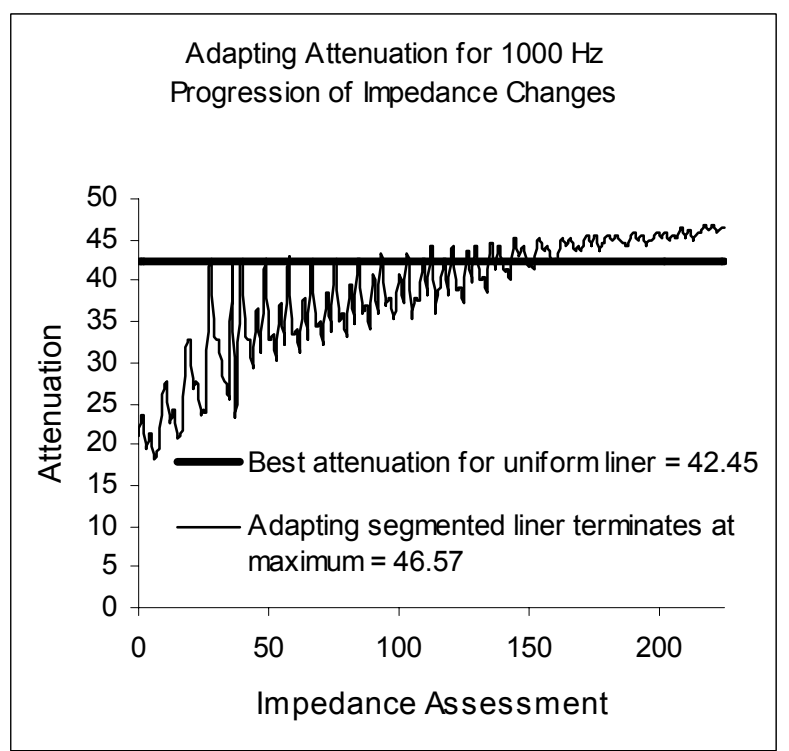

Fig. 7. Adaptation progression for segmented liner at $1000 \mathrm{~Hz}$.

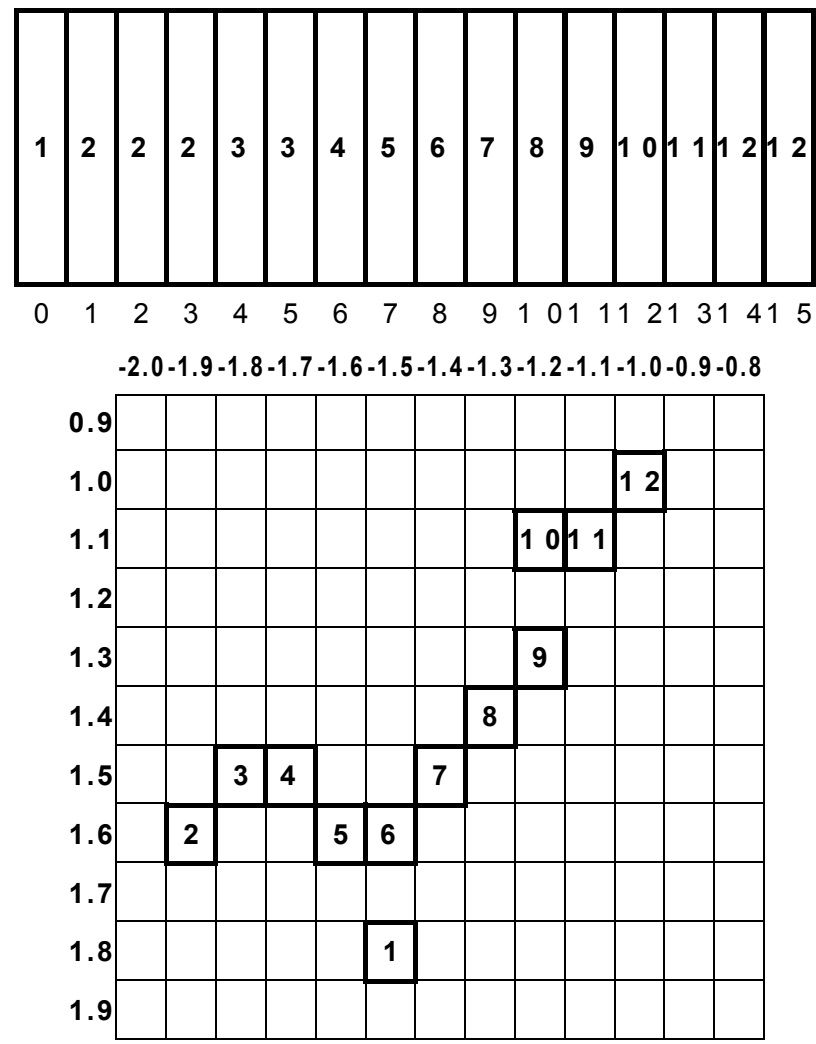

Fig. 8. Final segmented liner for $2000 \mathrm{~Hz}$. Global attenuation $=23.33 \mathrm{~dB}$.

Figs. 8 and 9 show the results of the simulation for $2000 \mathrm{~Hz}$. This results in a greater distribution of impedance values. The progression adapts to the attenuation of the best uniform liner quickly and then far surpasses it. 


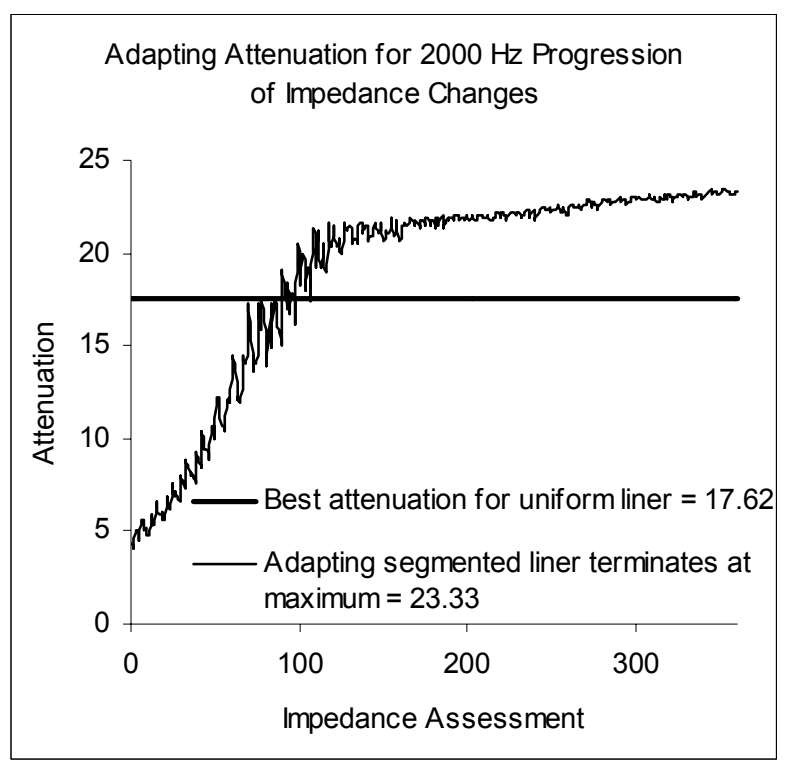

Fig. 9. Adaptation progression for segmented liner at $2000 \mathrm{~Hz}$.

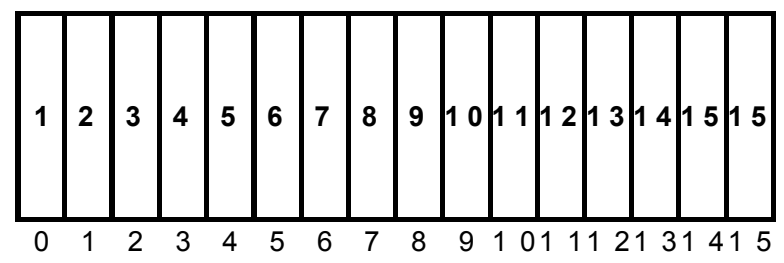

$-2.0-1.9-1.8-1.7-1.6-1.5-1.4-1.3-1.2-1.1-1.0-0.9$

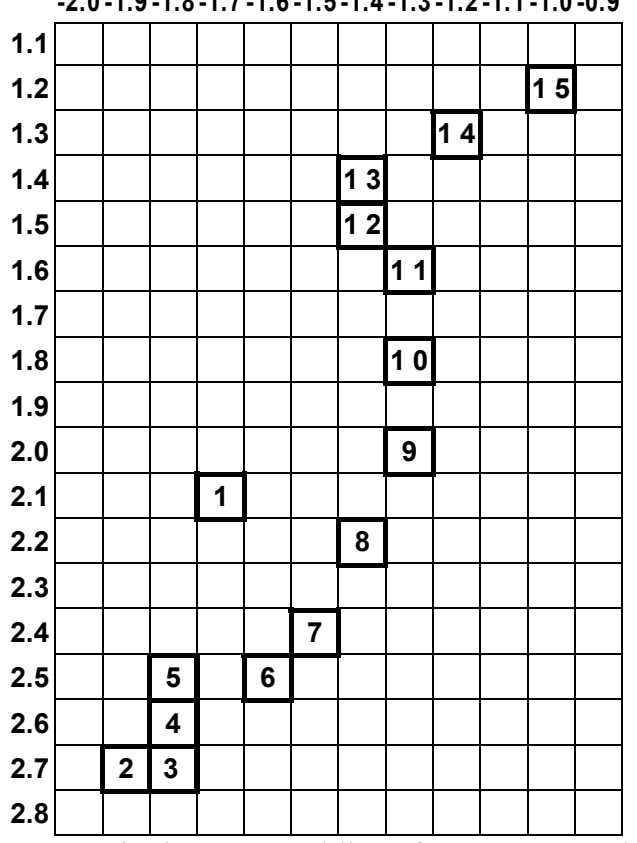

Fig. 10. Final segmented liner for $2500 \mathrm{~Hz}$. Global attenuation $=17.33 \mathrm{~dB}$.

Figs. 10 and 11 show the results of the simulation for $2500 \mathrm{~Hz}$. This displays the widest distribution of impedances and the largest number of impedances of all frequencies. As Fig. 11 depicts, the progression achieves attenuation for the best uniform liner after the first few segments freeze and continues to improve the attenuation thereafter.

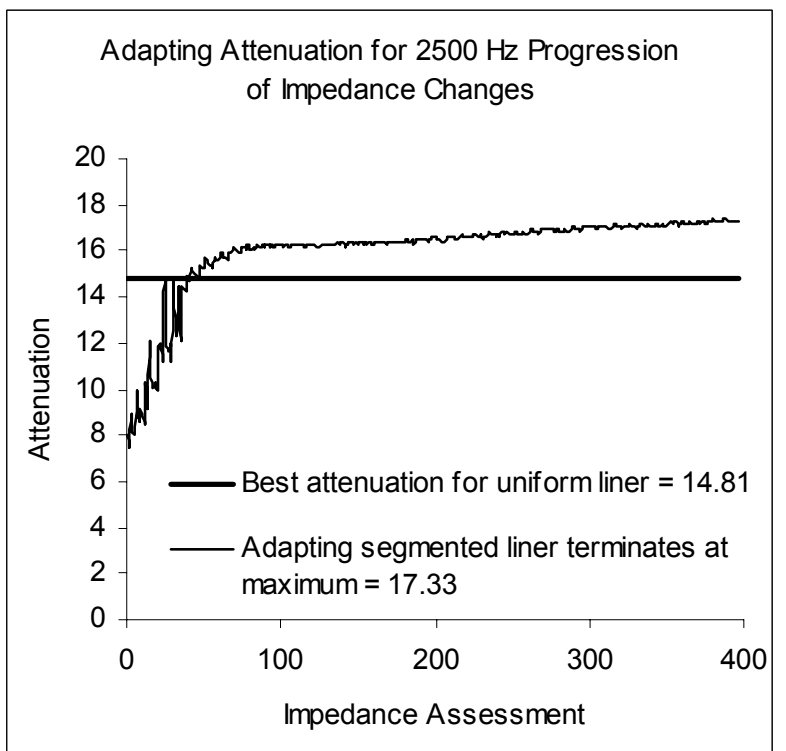

Fig. 11. Adaptation progression for segmented liner at $2500 \mathrm{~Hz}$.

Adapting Attenuation for 1500, 1000, 2000 and $2500 \mathrm{~Hz}$ Progression of Impedance Changes

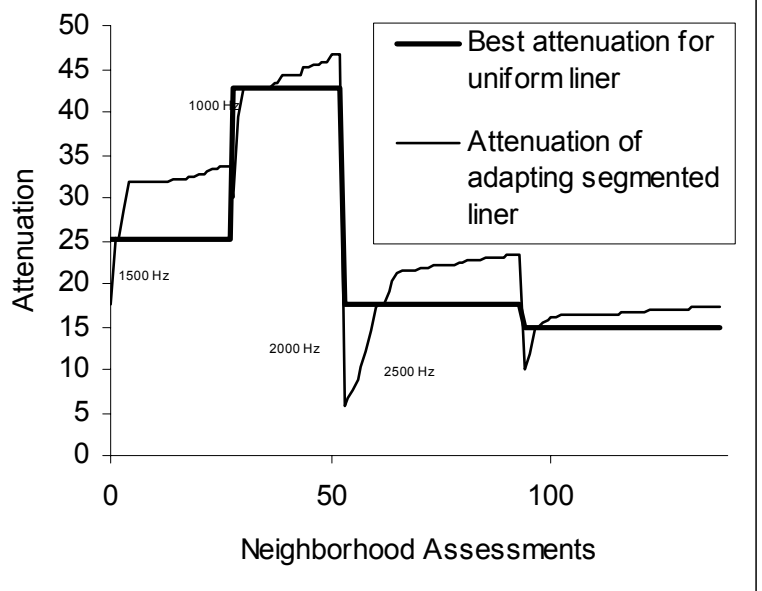

Fig. 12. Adaptation to best segmented liner for 4 frequencies.

Fig. 12 combines the adaptation process sequentially for the frequencies 1500,1000, 2000, and $2500 \mathrm{~Hz}$. This plot exemplifies the problem with a static, passive liner: there is no uniform liner that is optimal for all frequencies and no uniform liner that provides the level of noise reduction that can be attained with an adaptable liner (e.g., a duct with the best uniform liner for $1000 \mathrm{~Hz}$, when faced with a change in frequency to $2000 \mathrm{~Hz}$, reduces the global attenuation from $42.7 \mathrm{~dB}$ to $4.78 \mathrm{~dB}$ ). Furthermore, it shows that a segmented liner is more effective in this instance than a uniform liner as, most of the time 
throughout the sequence, the global attenuation is higher than could be attained by a uniform liner. The times when the global attenuation falls below the best for a uniform liner are minimal correction periods. Fig. 12 is somewhat unrealistic as it depicts a change in frequency immediately following attainment of the best segmented liner for the previous frequency. In flight, the process for determining the best segmented liner for takeoff would take seconds followed by minutes of climbing, during which the acoustic signature remains relatively stable. During the transition from takeoff to cruise, the adaptation would occur in seconds followed by a much longer period of stability during cruise. The process could be configured so that it would only restart when the attenuation fell below a threshold.

Communication requirements of this approach are simple. A sensor is assumed that provides the acoustic power level for each segment. A computation and communication node (CCN) is allocated for each segment. The leader segment's CCN performs a series of impedance neighborhood assessments requiring only requests and responses for the acoustic power level of the exit segment. This request and response could be accomplished by a multi-hop route across the segments, but as the distance is relatively short (at maximum, the length of the liner), this could be a point-to-point broadcast. For each impedance change, the neighborhood assessment of the leader segment broadcasts the value to resonators of the leader segment and all slave segments. Communication is not required with frozen segments.

The method described could be used in the design of a nacelle to predetermine a table of liner definitions for anticipated acoustic conditions. Simplistically, three liner definitions could be predetermined for an aircraft in takeoff, cruise, and landing conditions. A centralized computer could determine the change in conditions, use the table to determine impedance definitions for that condition, and dictate these values to liner resonators. This would address the challenge that acoustic conditions change over the flight regime, but would not address other challenges: that theoretical characteristics of the design may not be met in the construction of the liner material or physical changes in the nacelle over time may change its absorption properties. It would also not account for other environmental factors such as atmospheric conditions. The methods described here can accomplish the task by adapting the liner impedance in real time through collection of relatively little information from a local view.

\section{Conclusion}

Research in wireless sensor networks has accelerated rapidly in recent years. The promise of ubiquitous control of the physical environment opens the way for new applications that will redefine the way we live and work. Due to the small size and low cost of sensor devices, visionaries promise smart systems enabled by deployment of massive numbers of sensors working in concert. To date, most of the research effort has concentrated on forming ad hoc networks under centralized control, which is not scalable to massive deployments.

In this research, an alternate approach is proposed based on models inspired by biological systems and a significant result is reported based on this new approach. This perspective views sensor devices as autonomous organisms in a community interacting as part of an ecosystem rather than as nodes in a computing network. The networks that result from this design make local decisions based on local information resulting in achievement of global goals. This represents engineering for emergent behavior in wireless sensor networks.

Our research recognizes that the sense and send, centralized architecture severely limits the potential of wireless sensor networks. Instead, we developed an alternate, more versatile view of a sensor network. A wireless sensor mote is an autonomous entity containing the ability to sense the environment, compute, remember, and communicate with its immediate neighboring motes. Limiting use of the sensor mote to sense and send data to a central controller or to route data from a neighboring mote toward the central controller is merely a choice of the designer. Alternatively, we view these autonomous motes as organisms dispersed within the environment to form an ecosystem. As autonomous organisms, they are free to make their own decisions based on local observations shared only with neighboring motes. As neighborhoods iterate in this process of making local observations and taking local actions, a global behavior emerges. Our challenge was to develop an algorithm specifying the iterations of local observations and actions which result in desired global behavior specifically to facilitate adaptive noise reduction in aircraft nacelles.

Current technology for noise abatement in aircraft engines is dependent on acoustic liners composed of static, passive resonators of uniform impedance. New technology allows the impedance of resonators to be modified in situ permitting the adaptation of the liner impedance to changing acoustic conditions. Furthermore, research has determined that a liner of uniform impedance does not provide the optimal noise abatement. This research addresses the issue of how to dynamically determine optimal segment impedances in situ as acoustic conditions change.

An algorithm has been presented whereby an optimal segmented liner is determined for changing acoustic conditions using only local and limited cooperation among communal segments. The optimal impedance for segments emerges from local information and cooperation. This eliminates single point of failure and other problems created by a centrally controlled architecture. Superior impedance patterns emerge that are not easily determined by prior design methods.

This research is just beginning and there are recognized limitations of the work to date. Sound input was limited to single frequencies and analysis methods were assumptive such that they are not simulating a full 
acoustic environment of aircraft engine nacelles in flight. Also, the full sensing, communication, and computation of the process has not been simulated nor analyzed for efficiency. While it is intuitively obvious that the proposed method has advantages over a centralized approach, analysis has not been done comparing relative efficiencies. Still, nothing is apparent that this process will not work in a real environment and provide the advantages anticipated. Our next steps are to improve the simulation to more accurately model the environment and compare the advantages with the centralized approach.

\section{References}

[1] B. Warneke, M. Last, B. Leibowitz, and K. Pister, SmartDust: communicating with a cubic-millimeter computer, IEEE Computer, vol. 34, no.1, pp. 44-55, 2001.

[2] National Research Council, Embedded Everywhere, National Academy Press, 2001.

[3] Jones, K., Lodding, K., Olariu, S., Wilson, L., and Xin, C., Biology Inspired Approach for Communal Behavior in Sensor Networks, presented at Minitrack on Wireless Sensor Networks and Applications, Proceedings of 39th Hawaii International Conference on System Sciences (HICSS-39), Kauai, Hawaii, January 4-7, 2006.

[4] K. Jones, K. Lodding, S. Olariu, A. Wadaa, L. Wilson, and M. Eltoweissy, Biomimetic Models for Wireless Sensor Networks," In Handbook of Bioinspired Algorithms And Applications, S. Olariu, and A. Zomaya, Eds., Chapman \& Hall/CRC Computer \& Information Science, Boca Raton, Florida, USA, 2004, ISBN 1-58488475-4.

[5] Helmholtz, H. and Ellis, A., On the Sensations of Tone as a Physiological Basis for the Theory of Music, Dover Publications, New York, 1954.

[6] Mani, R., Acoustic Duct with Peripherally Segmented Acoustic Treatment, U.S. Patent 3,937,590, February, 1976.

[7] Watson, W., Robinson, J., Jones, M., and Parrott, T., Design and Attenuation Properties of Periodic Checkerboard Liners, AIAA-2003-3309, 9th AIAA/CEAS Aeroacoustics Conference and Exhibit, Hilton Head, South Carolina, May 12-14, 2003.

[8] De Bedout, J., Franchek, M., Bernhard, R., and Mongeau, L., Adaptive-passive Noise Control With Selftuning Helmholtz Resonators, Journal of Sound and Vibration, Vol. 202(1), pp. 109-123, 1997.

[9] Nark, D. Farassat, F., Pope, D., Vatsa, V., The Development of the Ducted Fan Noise Propagation and Radiation Code CDUCT-LaRC, AIAA Paper 2003-3242, 2003. 